\title{
Metal-Assisted Chemical Etching of Silicon 3D Nanostructure Using Direct Electric Field
}

\author{
Jiao Xiaodong*, Chao Yan, Wu Liqun, Yao Anqi \\ School of Mechanical Engineering, Hangzhou Dianzi University, Hangzhou 310018, China
}

\begin{abstract}
Keywords: noble metal; 3D nanostructure; direct electric field; controllable fabrication.
Abstract: Metal-assisted chemical etching (MaCE) of silicon ( $\mathrm{Si}$ ) is a well-used method for the fabrication of Si nanostructures. To simplify the control etching for the fabrication of $\mathrm{Si} 3 \mathrm{D}$ nanostructures, we developed a new method using direct electric field to control the etching direction. We examined the MaCE process in the electric field, and evaluated the effect of different electric field frequencies on the production of $\mathrm{Si}$ nanostructures. The results demonstrate that electric fields can effectively control etching direction and can be used to fabricate Si 3D nanostructures. Optimization of the electric current density and electric field frequency range has been performed.
\end{abstract}

\section{Introduction}

Silicon nanostructures exhibit promising application potentials in the fields of opto-electronics, biochemical sensors, Micro and NanoElectroMechanical Systems, energy conversion, and energy storage $^{[1]}$. Controlllable fabrication of $\mathrm{Si}$ nanostructures is a prerequisite for their application. Various methods can be used to fabricate Si nanostructures, including vapor-liquid-solid (VLS), reactive ion etching (RIE), electron beam lithography (EBL) and metal-assisted chemical etching (MaCE). Fabricating complex 2D and 3D geometry is challenging for all of these techniques, however, and can be cost-prohibitive ${ }^{[2]}$. Metal-assisted chemical etching has attracted increasing attention in recent years because it allows cost-efficienct fabrication of Si nanostructures with high feature fidelity and high aspect ratio.

In MaCE, a metal catalyst is used to locally increase the silicon dissolustion rate in a solution containing hydrofluoric acid and an oxidizing agent by creating a localized galvanic cell arround the metal catalyst particle. The etching process starts as $\mathrm{H}_{2} \mathrm{O}_{2}$ is catalytically reduced on the metal catalyst to form a local cathode which consumes the electrons from silicon atoms. Silicon atoms are oxidized to $\mathrm{Si}^{4+}$ or $\mathrm{Si}^{2+}$ with the reaction continuing as the metal catalyst travels into substrate as the silicon around and beneath the metal catalyst is dissolved. Obviously, the movement track of the metal catalyst determines the structure in $\mathrm{MaCE}$, and $\mathrm{H}_{2} \mathrm{O}_{2}$ affects the etching rate, direction, and feature fidelity. In addition, the etchant compoition, crystallographic orientation, metal catalyst material and etching temperature can also affect the etching direction ${ }^{[3-5]}$. The ability of controlling the etching direction is critical for fabricating complex 2D and 3D nanostructures and fabricating real-world devices for scientific and industrial uses. Numerous articles report strategies for control of the etching direction by limiting the motion of the metal catalyst ${ }^{[5-9]}$. Owen et al ${ }^{[8]}$ report a new method of fabricating complex 3D nanostrutures with high feature fidelity using a shaped metal catalyst in MaCE. Others have reported that an etchant with different molarity ratio of $\mathrm{H}_{2} \mathrm{O}_{2}$ to $\mathrm{HF}$ can affect the movement direction of metal catalyst as well as etching direction ${ }^{[3,5,10,11]}$. In an etchant with a high $[\mathrm{HF}] /\left[\mathrm{H}_{2} \mathrm{O}_{2}\right]$ ratio, a $\mathrm{Si}(111)$ wafer is etched along $<100>$ direction. When the ratio is sufficiently low, etching direction proceeds along the $<111>\operatorname{direction}^{[3]}$. The back bond strength and the surface oxidation atate of $\mathrm{Si}$ contribute to the etching direction, among other factors, but $<100>$ direction is the preferential etching direction of Si. Much research has been dedicated to control of the etching direction to allow fabrication of silicon nanowires ${ }^{[12-14]}$, precise control of etching directions remains elusive.

This manuscript introduces both the method to fabricate complex 3D nanostrutures and a new hypothesis for controling the motion of metal catalyst in MaCE by using electric field. Direct electric field supplies sufficient driving force for metal catalyst motion, allowing control of the etching direction. These results shed new light on the topic of metal catalyst motion in MaCE and 
extend the electrophoretic model proposed by Peng et $\mathrm{al}^{[15]}$. In this article, the first section verifies the feasibility of controlling etching direction using electric field and proposes an electric field control model. This model details the mechanism of controlling etching direction in the electric field and evaluates the effects of different frequencies on the $\mathrm{Si}$ nanostructures. The results demonstrate that electric field can effectively control etching direction and can be used to fabricate Si 3D nanostructures. This method represents a new paradigm in etching and a new scenario for fabrication of 3D nanostructures.

\section{Experimental}

N-type silicon (111) wafers with the thickness of $2 \mathrm{~mm}$ were used with resistance of $5 \Omega \cdot \mathrm{cm}$. The silicon wafers were cut into $2 \mathrm{~cm} \times 2 \mathrm{~cm}$ pieces. A couple of graphite electrodes $(2 \mathrm{~cm} \times 2 \mathrm{~mm})$ were pasted to the side of silicon wafers so that electric field could pass through the silicon side. Prior to each experiment, the samples were cleaned with standard RCA. Silicon pieces with a couple of graphite electrodes were put into an etching solution contained in a reactive vessel. Another couple of graphite electrodes were placed on both ends of the reactor so that electric field could pass through silicon front side (Fig. 1.). Two couple of graphite electrodes connected two current controllers. Therefore, two electric fields of different direction were perpendicular to the side and front surfaces of the silicon wafer. The etching solution was $7.2 \mathrm{Mol} / \mathrm{L} \mathrm{HF}, 0.3 \mathrm{Mol} / \mathrm{L} \mathrm{H}_{2} \mathrm{O}_{2}$ and $0.01 \mathrm{Mol} / \mathrm{L} \mathrm{AgNO}_{3}$. After etching, the silicon piece was dipped into an aqueous solution of $\mathrm{HNO}_{3}$ $(30 \% \mathrm{~W} / \mathrm{W})$ and then rinsed with de-ionized water to remove any residual silver.

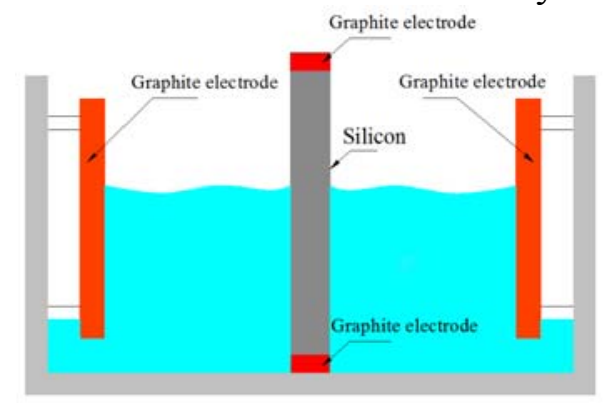

Fig. 1. Experimental device.

\subsection{Fabrication of Si nanostructure using electric field in MaCE}

All experiments were carried out at room temperature. The power of electric fields were placed on both ends of the reactor so electric field lines are perpendicular to the front wafer surface. The current density of the electric field was maintained at $1.5 \mathrm{~mA} / \mathrm{cm}^{2}$. Fig. 2 show a cross-sectional SEM image of the $<111>$ Si nanowires (SiNWs) on Si (111). As expected, Si nanowires arrays were produced using electric field in MaCE. To verify whether the electric field can affect the fabrication of SiNWs, we also performed an experiment by increasing the current density of the electric field to $4.5 \mathrm{~mA} / \mathrm{cm}^{2}$. The result is shown in Fig. 3. There are not SiNWs fabricated on Si (111). In contrast, a bump nanostructure is produced on $\mathrm{Si}$ (111).

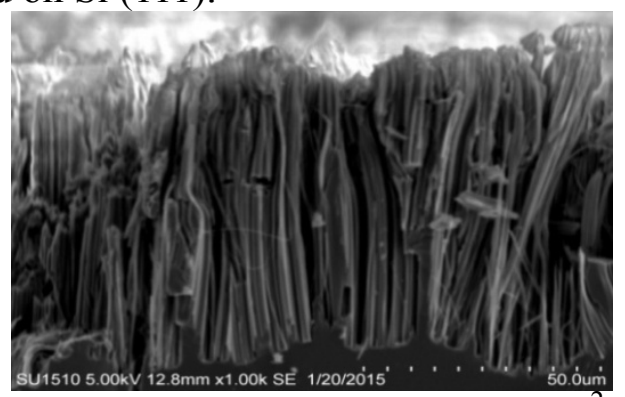

Fig. 2. Results using an electric current density of $\mathrm{J} 1=1.5 \mathrm{~mA} / \mathrm{cm}^{2}$, cross-sectional SEM image of the $<111>$ Si nanowires on $\operatorname{Si}(111)$. 


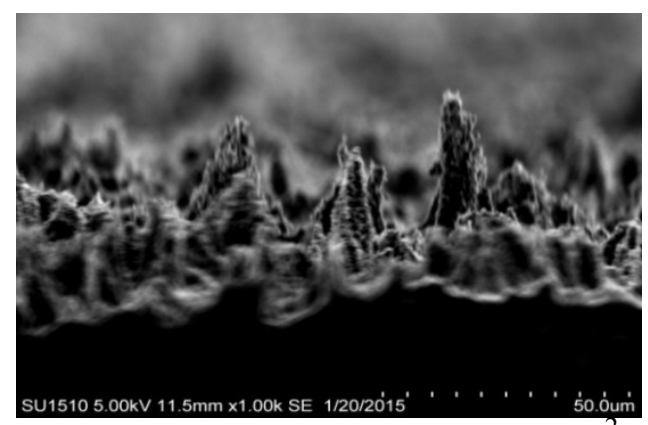

Fig. 3. Results using an electric current density of $\mathrm{J}_{2}=4.5 \mathrm{~mA} / \mathrm{cm}^{2}$, cross-sectional SEM image of bump nanostructure on $\mathrm{Si}$ (111).

From the above two experiments, we can deduce how electric field strength affects the geometry on silicon. The etched structure in MaCE can be viewed as the movement track of the metal catalyst, and therefore controlling motion of metal catalyst is the key to control of the etching direction. Peng et al report an electrokinetic model and propose that the self-generated electric field supplies the main driving force for metal catalyst motion. The electrophoretic slip velocity of metal catalyst particle in this model is described by ${ }^{[15]}$ :

$v_{e p}=\frac{2 \varepsilon \zeta E_{/ /}}{3 \eta}$

Where $v_{e p}, \varepsilon, \zeta, E_{/ /}$and $\eta$ are the slip velocity of metal catalyst, the permittivity of the solution, the zeta potential of the metal catalyst nanoparticle, self-generated electric field, and the viscosity of the solution, respectively.

The physical mechanisms driving metal catalyst motion are not very well understood, but our experimental results show that electric field does effect the geometry on silicon. Hochbaum et al ${ }^{[16]}$ also report that the etching of $\mathrm{Si}$ in $\mathrm{MaCE}$ is likely to follow an electrochemical mechanism, similar to what occurs during conventional electrochemical synthesis of porous $\mathrm{Si}$. The major difference between $\mathrm{MaCE}$ and electrochemical previous porous Si synthesis is that the current flux inducing porosification in $\mathrm{MaCE}$ is provided by continuous $\mathrm{Ag}^{+}$reduction from solution, while that in electrochemical porous Si synthesis is driven by an applied bias through back electrical contacts. High current density of the electric field damaged the etching mechanism of MaCE, preventing fabrication of the Si nanowires. The results of our experiments support the findings of $\mathrm{Hu}$ et $\mathrm{al}^{[12]}$ who report that high current density of the electric field can fabricate porous silicon and low current density of the electric field can fabricate silicon nanowires. Therefore, appropriate current density is required to fabricate nanostructure. In our experiments, the optimization range of the electric current density is $\mathrm{J}<4.0 \mathrm{~mA} / \mathrm{cm}^{2}$ and the optimization electric current density is $1.5 \mathrm{~mA} / \mathrm{cm}^{2}$.

\subsection{Electric field control etching direction}

The electrokinetic model presented by Peng et $\mathrm{al}^{[15]}$, is a force for metal catalyst motion. This model is rooted in the observation that the electrochemical reactions of $\mathrm{H}_{2} \mathrm{O}_{2}$ reduction and $\mathrm{Si}$ oxidation in MaCE closely mirror $\mathrm{H}_{2} \mathrm{O}_{2}$ reduction and oxidation reactions that drive the autocatalytic electrophoretic motion of metal catalyst in an $\mathrm{H}_{2} \mathrm{O}_{2}$ solution detailed by Paxton et al ${ }^{[17]}$. In MaCE, Ag particle surface would be negatively charged and, at the same time, the difference in conductance of electrons and hydrogen ions creates a strong self-generated electric field. Self-generated electric field supplys sufficient driving force for negatively charged Ag particles. Obviously, the self-generated electric field is different to control. We added out electric field to replace self-generated electric field and control etching direction by using out electric field. To explore controlling etching direction and to verify that electric field has the ability to control the motion of metal catalyst to fabricate 3D nanostrutures, we performed an experiment using two different directions of electric field. Fig. 4 shows that the motion direction of metal catalyst particle is controlled by the direction of electric field. Different electric fields alters direction of the metal catalyst. Controlling the electric field's direction and etching time can fabricate specified structures on silicon. The open lateral electric field and vertical electric field must be applied synchronously. 
We used a current density of the electric field of $1.5 \mathrm{~mA} / \mathrm{cm}^{2}$. After etching for 60 minutes, we removed the electric fields and then continued etching for 20min. Fig. 5 shows the result. We can see that arc-like nanostructure is produced on silicon.
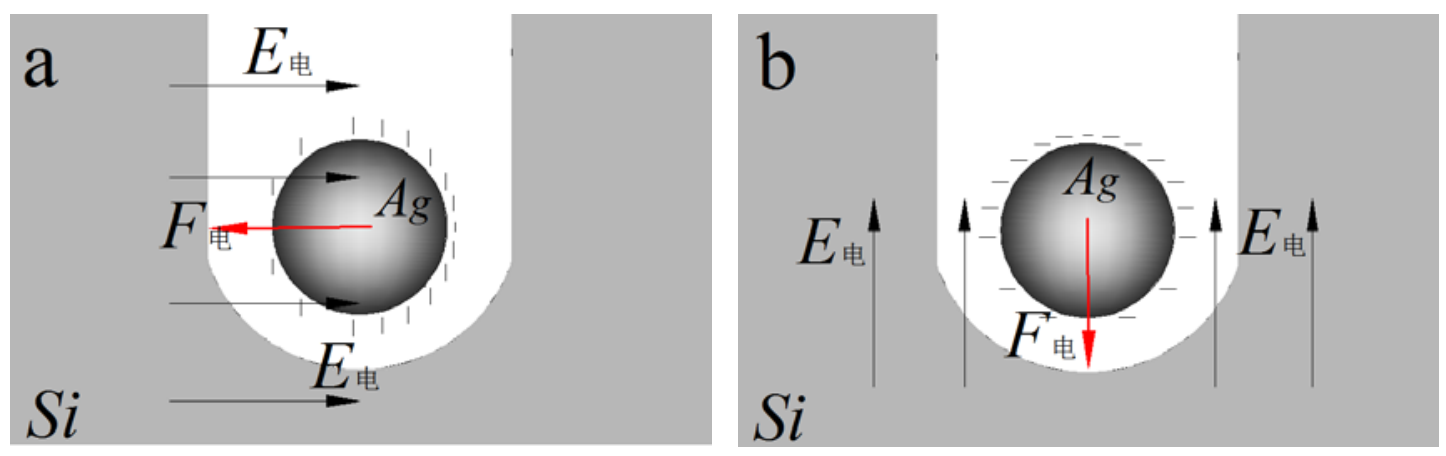

Fig. 4. Lateral electric field control motion of metal particle. Vertical electric field control motion of metal particle.

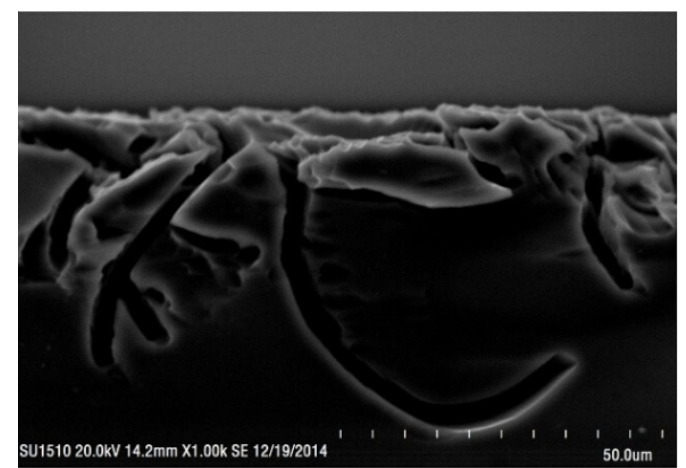

Fig. 5. Cross-sectional SEM image of nanostructure on Si (111).

\section{Conclusions}

In this papper, we have investigated the process of controling etching direction, and propose a new method to fabricate 3D nanostructures by using direct electric field. An electric field control model has been formulated to study the mechanism of MaCE in the electric field. This model has the ability to control the motion of metal catalyst and allow the controllable motion of metal catalyst in a composite electric field. High current density of the electric field does not allow fabrication of silicon nanowires, but reasonable current density is helpful to control etching direction to fabricate nanostructures. The optimal range of the electric current density was $\mathrm{J}<4.0 \mathrm{~mA} / \mathrm{cm} 2$, and the optimal electric current density was $1.5 \mathrm{~mA} / \mathrm{cm} 2$. This process is in the early stages of development, and further work is needed to more fully understand etching mechanics. We hope to both demonstrate the potential for MaCE by using electric field to control etching direction but also inspire others to continue investigation of this promising process.

\section{Acknowledgements}

This work was supported by the National Natural Science Foundation of China(51175134);By Key Zhejiang Provincial Natural Science Foundation of China(LZ15E050004);

\section{References}

[1] Chan C K, Peng H, Liu G, et al. 2008, 3(1): 31-35.

[2] Huang Z, Geyer N, Werner P, et al. Advanced Materials. 2011, 23(2): 285-308.

[3] Huang Z, Shimizu T, Senz S, et al. The Journal of Physical Chemistry C. 2010, 114(24): 10683-10690. 
[4] Zhang M, Peng K, Fan X, et al. JOURNAL OF PHYSICAL CHEMISTRY C. 2008, 112(12): 4444-4450.

[5] Chen H, Wang H, Zhang X, et al. NANO LETTERS. 2010, 10(3): 864-868.

[6] Cichoszewski J, Reuter M, Schwerdt F, et al. Electrochimica Acta. 2013, 109: 333-339.

[7] Sivakov V A, Broenstrup G, Pecz B, et al. JOURNAL OF PHYSICAL CHEMISTRY C. 2010, 114(9): 3798-3803.

[8] Hildreth O J, Lin W, Wong C P. ACS Nano. 2009, 3(12): 4033-4042.

[9] Hildreth O J, Rykaczewski K, Fedorov A G, et al. NANOSCALE. 2013, 5(3): 961-970.

[10] Chartier C, Bastide S, Levy-Clement C. ELECTROCHIMICA ACTA. 2008, 53(17): 5509-5516.

[11] Liu K, Qu S, Zhang X, et al. JOURNAL OF MATERIALS SCIENCE. 2013, 48(4): 1755-1762.

[12] Hu Y, Peng K, Liu L, et al. SCIENTIFIC REPORTS. 2014, 4(3667).

[13] Hu Y, Peng K, Qiao Z, et al. NANO LETTERS. 2014, 14(8): 4212-4219.

[14] Liu L, Peng K, Hu Y, et al. ADVANCED MATERIALS. 2014, 26(9): 1410-1413.

[15] Peng K, Lu A, Zhang R, et al. Advanced Functional Materials. 2008, 18(19): 3026-3035.

[16] Hochbaum A I, Gargas D, Hwang Y J, et al. NANO LETTERS. 2009, 9(10): 3550-3554.

[17] Paxton W F, Baker P T, Kline T R, et al. JOURNAL OF THE AMERICAN CHEMICAL SOCIETY. 2006, 128(46): 14881-14888. 